\title{
UNA NOTA SOBRE LA LENGUA, EL DERECHO Y LA SOCIEDAD
}

Alberto Escobar

1. Esta nota ensayará presentar dos dimensiones del tratamiento de la lengua, en el texto de la Constitución vigente en el Perú. Como es conocido, nuestra carta del 79 aborda en tres articulos: el 83, el 35 y el 34, asuntos que se relacionan con las lenguas habladas dentro del Estado peruano (1). Sin embargo, es mi intención presentar, brevemente, el marco histórico del pensamiento social, dentro del cual hay que situar la evolución que antecede a los valores que signan las actitudes y las normas del derecho.

(1) Art. 830- El castellano es el idioma oficial de la República, También son de uso oficial el quechua y el aymara en las zonas y la forma que $l_{a}$ ley establece. Las demás lenguas aborigenes integran asimismo el patrimonio cultural de la nación.

Art. 35- El Estado promueve el estudio y conocimiento de las lenguas aborigenes. Garantiza el derecho de las comunidades quechua, aymara y demás comunidades nativas a recibir educación primaria en su propio idioma o lengua.

Art. 34- El Estado preserva y estimula las manifestaciones de las culturas nativas, asi como las peculiares y genuinas del folklore nacional, el arte popular y la artesania. 
En otras palabras, me propongo relacionar el pensamiento que sustenta los citados artículos con las teorias sociales y con las posiciones de derecho, que son sus antecedentes dentro de nuestro proceso cultural y social.

2. Después de la debacle de la guerra del Pacífico, Manuel González Prada (1848-1918) se preguntó insistentemente por las causas de ese descalabro nacional. Para los estudiosos del pensamiento peruano en general, como para los que se interrogan sobre la evolución del pensamiento politico en nuestro pais (2), el meollo de la preocupación que agobiaba a González Prada residia en la comprobación de que el Perú no haba llegado a configurar un Estado-Nación, como si lo habia logrado Chile. Es fácil darsc cuenta de estas inferencias, tanto en el discurso pronunciado en el teatro Politeama en 1888, como en el articulo "Nuestros Ind'os" de 1904 y en una serie de pasajes de la obra en prosa del célebre escritor. Por ejemplo, en el siguiente:

"No forman el verdadero Peri las agrupaciones de criollos i estranjeros que habitan la faja de tierra situada entre cl Pacifico i los Andes; la nación cstá formada por las muchedumbres de indios diseminados en la banda oriental de la cordillera. T'rescientos años há que el indio rastrea en las capas inferiores de la civilización, siendo un híbrido con los ricios del bárbaro i sin las virtudes del europeo: enseñadle siquiera a leer $i$ escrib:r, $i$ veréis si en un cuarto de siglo se levanta o no a la dignidad de hombre. A vosotros, maestros d'escuela, toca galvanizar una raza que se adormece bajo la tirania del juez de paz, del gobernador i del cura, esa trinidad embrutecedora del indio". En: Pájinas Libres, p. 67.

(2) Véase la Historia dz las ideas en el Perú contemporáneo. 2ts. (1965). de Augusto Salazar Bondy, y el vol. XI de la Historia del Perú (1980) que contiene el ensayo "Las ideas en el Perú contemporáneo" (pp. 113-415), por David Sobrevilla. Cf. especialmente el balance sobre el pesitivismo y las posiciones de González Prada y Manuel V. Villarán eal contraste con las preconizadas por Javier Prado y Mariano H. Cornejo (pp. 155-157). La perspectiva de Cotler (1978) sitúa el cutramado histćrico-social de las ideas que afloran en torno del concepto Estr. do-nación, del papel del indio y del indigenismo y de la descriminación social en favor del blanco y de su emblemática. Bonilla et al. (2a ed. 1981) dicen que la repúbica se ha basado en la igualdad universal y en lo desiguaìdad social como convicción. 
Este pensamiento está intimamente eslabonado a uno que aparece en el mismo texto, páginas adelante, y que contrasta dos fenómenos que en muchos conceptos son contradictorios: "Con las muchedumbres libres aunque indisciplinadas de la Revolución, Francia marchó a la victoria; con los ejércitos de indios discipl:nados y sin libertad, el Perú irá siempre a la derrota. Si del indio hicimos un s'ervo ¿qué patria defenderá? Como el siervo de la Edad media, sólo combatirá por el señor feudal". (Idem p. 65).

Sólo quisiera mencionar que los enjuiciamientos de González Prada - como lo ha hecho notar Bruno Podestá-, están en las bases programáticas de la "Declaración de Principios" del partido Unión Nacional, hacia 1891, en los que se establece que en nuestro pais: ". . conviene de una manera especial unir a los hombres por el vínculo de las ideas, tender a la formación del espiritu nacional, fomentar verdadera solidaridad de intereses entre la costa y la sierra" (3). Por eso tiene importancia subrayar, como lo apunta Podestá, que antes de su derivación anarquista, la matriz del pensamiento Gonzálezpradiano se ligaba al proyecto que años antes intentó levantar el civilismo, y por el cual, se concebia el pais -como hoy en dia mucha gente lo hace aún-. a través de una oposición entre la costa (occidental y civilizada) y la sierra (aborigen y atrasada). En consecuencia el proyecto nacional que seguia era el de fundir, reunir ambas regiones, ambos compartimientos y a sus hombres, y esta acción se entendia como "el peruanizar" nuestra soc'edad y nuestro país; el "integrarlos". Lo anterior es importante pues no hay duda que el pensamiento que adquirió brillo en el verbo y en la prosa de González Prada, contribuyó a definir dos momentos fundamentales en lo que se ha llamado la generación del Novecientos y la genera ción del Centenario. Sin ánimo de extenderme demasiado sobre cste punto, creo que es menester insistir en que persiste una especie de diálogo (o coloqulo) ininterrumpido, que pasa por las obras de los niembros de estas generaciones: Francisco García

(3) Además de "La gestación del primer indigenismo: el caso González Prada" (1980), vale leer los trabajos de Pcdestá sobre el mismo pensador, publicados en 1975, 1978. Garcia Salvattecci (1972?) traza, as: mismo, una revisión inteligente. Para la feudalidad andina, cf. Burga $y$ Flores Galindo (1979). 
Calderón, Victor Andrés Belaúnde, José de la Riva Agüero, para dar hitos de la generación del Novecientos; y Haya de la Torre, Basadre, Porras, Mariátegui, Sánchez, para indicar referenkias a la generación del Centenario. Pues bien, lo que trato de explicar es que, desde hace muchas décadas se tiene conciencia que hay un problema de identidad nacional, para muchos de los habitantes que juridicamente forman parte de la república peruana. No es el caso de ponerse a inventariar cuáles fueron las salidas imaginadas por los miembros de la generación aristocrática, o cuáles fueron las posiciones que condieron, a la luz del establecimiento de la conducción politica de Haya y de Mariátegui. Creo que los interesados en este punto harán bien en dirigir su mirada a algunos estudios que se han hechos públicos en los últimos 10 años, en los cuales se revisan todos estos enfoques y se pasa atento escrutinio, a los distintos cauces de interpretación que se han trajinado en los replanteamientos de la historia social y de la ciencia política en el Perú y en América (4). Por el momento invocaré un juicio de Salazar Bondy, para quién, a pesar de que González Prada en su tiempo era visto como un radial a ultranza y cuyo balance sobre la sociedad no era muy favorable, en opinión de Salazar Bondy, el autor de Pájinas Libres resulta que tenía una imagen menos sombría que la de Alejandro Deustua (1849-1945), el célebre maestro de varias generaciones y quizás una de las figuras nacionales más reputadas en la incitación al reflexionar filosófico en el Perú. En efecto, para Deustua eran descalificables en la vida peruana tanto el indio como el obrero, porque eran incapaces de salvarse a si mismos, pero también toda la clase dirigente peruana, que estaba desquiciada (5). De modo que, según Deustua, no era posible que se avizorara ninguna salvación, sin la presencia de una élite conductora del pais, y que ésta fuera una minoría selecta, capaz de sacudirse del fardo de las tradiciones y de los hábitos nocivos acumulados durante

(4) Además de los nombres anteriores, cf. François Bourricaud, Poder y sociedad è el Perá contemporáneo (1967). A. Quijano. Imperialismo, clases sociales y estado en el Perú 1890-1930 (1978). Bonilla (1974) sostiene que el civilismo era la traducción ideológica del resentimiento por la pérdida del guano, incapaz de ser una reivindicación nacional.

(5) "La imagen del Perú que surge de esta auscultación es quizás la más sombria y pesimista de toda la critica nacional". (Salazar, op. cit., p. 188). 
años. "Acceder al nivel de la moralidad y la cultura y llevar a ese nivel a las promociones de los futuros conductores, es tarea de moralización encomendada a los reformadores del Perú", p. 189. Es por eso que, para Salazar, el debate pedagógico se instala como un momento decisivo a lo largo del proceso ideológico peruano. $Y$, en lo que a nosotros respecta, es pertinente sef̃alar que, tanto en la comprensión de la sociedad peruana, así como de al crisis de identidad que la ha aquejado, es menester insertar $y$ comprender el problema de la lengua, y las diversas actitudes que frente a él han tenido los distintos componentes politicos y las clases sociales (o varios segmentos de clases), según algunos pasos de nuestra vida republicana.

3. En virtud de un principio jurídico que confiere a la Carta magna prioridad sobre las otras disposiciones legales del pais, la Constitución del 79 ha derogado al decreto 21156 de mayo de 1975. Como es sabido, fue ese decreto ley, el que oficializó la lengua quechua en paridad de derechos con la lengua castellana, dentro del ámbito en toda la república. Por esa razón no es antojadizo anotar que en 1975 "se oficializó" el quechua y que en 1979 "se le desoficializó". Tiene interés mencionar que la oficialización del quechua estaba en relación con una serie de medidas, las que tendian a presentar sesgos anti-tradicionales en el diseño de un proyecto societal distinto del que había primado en el pais hasta 1968, y en especial con una diferente impronta de un proyecto educativo.

Por eso es muy útil recordar que el multilingüismo data de muchos siglos en lo que ahora es el Perú, incluso siglos antes de la llegada del hombre español. Tanto es así, que ya en épocas del incario existian 3 lenguas generales $y$, como lo ha probado Torero, fue por su familiaridad con el quechua, el aymara y el puquina, que el Virrey Toledo nombró traductor oficial del virreynato a González Holgu'n (6). Lo que importa es poner en evi-

(6) El volumen colectivo El reto del multilingüísmo en el Perú (1972) compilado por A. Escobar, ofrece en 10 contribuciones una adecuada presentación de la magnitud del desafío y de la complejidad de la respuesta. Cf. A. Torero El quechua y la historia social andina (1974) y A. Escobar "El problema de la lengua y la identidad nacional" en: Perú: identidad nacional. CEDEP (1979). A. Escobar y Matos Mar J., Alberti G. PERU ipais bilingüic? IEP, 1975. 
dencia que de entonces a la fecha, muchas cosas han cambiado en el pais; pero, que las pruebas aportadas por los investigadores nos reafirman, cada vez más en la convicción de que la nuestra es una sociedad multilingüe y pluricultural. En la fecha la movilidad interna y el desplazamiento de los habitantes dentro del Perú, así como los traslados del campo a la ciudad han llegado a tal magn'tud, que en el último censo se confirma lo que se anunciaba ya en el anterior; es decir, que el Perú actualmente tiene mayor proporción de habitantes en los centros urbanos y ya no en áreas rurales (7). Aparentemente este hecho aporta una diferente configuración de la distribución demográfica de la sociedad peruana, y podria entenderse que ha cambiado fundamentalmente los problemas inherentes a la lengua o las lenguas. No obstante lo dicho, podemos afirmar que la correlación entre los bilingües no es unilineal, de modo que se pueda señalar que la tasa de hablantes de español registrados en las cifras oficiales, supera la barrera comunicativa y derivados. Por esta causa insistimos en que es necesario considerar otra vez, desde otros puntos de vista, un planteo referente a las lenguas del pais y sus roles sociales (8).

4. Para hacerlo más breve, adelantaré que en el intento de 1975 se queria alcanzar politicamente un grado de coherencia

(7) En 1974. Escobar presentó en la Primera Conferencia Interamericana sobre la Educación Bilingüe un balance, a esa fecha, de problemas que encaraba dicha educación en referencia con la densidad demográfica, la oposición urbe/campo y la atención escolar, entre otros factores adversos. Cf. Escobar (1975). Los resultados provisionales del censo de población de 1981, permiten contrastar los datos recientes con los de 1972. En efecto, el año pasado la población general ascendia a $17^{\prime} \mathrm{de}$ habitantes, la urbana llegaba a 11' y la rural a casi 6'; en cambio eo 1972, la cifra general era $13.5^{\circ}$, la urbana de $8^{\circ}$, y la rural de casi $5.5^{\circ}$. Es necesario remontarse hasta 1961, cuando la población total sumaba $10^{\circ}$ para encontrar una ligera diferencia a favor del campo. Entonces el área urbana era de $4.6^{\circ}$ y la rural de $5.2^{\prime}$. De esa fecha el cambio de la proporción de la tasa de crecimiento urbano es creciente, pero sin que ese hecho cancele el problema designado como la cuestión de la lengua. Véase el análisis de la interrelación entre el fenómeno étnico y regional en el área andina, expuesto por Heraclio Bonilla (1982).

(8) Cf. Desarrollo y Educación en América Latina, vol. 2 (1981), y espepificamente entre los años 50 y 80 y la caracterización de las estructuras del sistema educativo latinoamericano. Véase A. Escobar (1972, 1976, 1978, 1981 y 1982). 
entre el objetivo perseguido y proyecciones de las medidas técnicas diseñadas para ser puestas en acción sucesivamente. (Durante dos años se adelantaron un juego de instrumentos y trabajos, en general preparatorios, y después del 78 , prácticamente se suspendió toda acción efectiva).

Tenemos la impresión que en 1979, o no se tenía conciencia de la barrera comunicativa entre los distintos tipos de hablantes del pais, ni de los tipos de castellano ni del efecto del multilingüismc que surge de la vigencia del pluriculturalismo de este pais; o, si lo habia, prevaleció el criterio de reconducir toda la cuestión de la lengua, que está ligada al problema de la identidad nacional, hacia los predios de la educación (sea regular o extraordinaria). Es decir, que se abrazaba nuevamente el estereotipo educativo, como si la educación fuera una variable independiente, aparte de los factores implicados y de las valoraciones que campean en el ámbito de las sociedades, en especial en sus estamentos directivos, políticos y burocráticos (9).

En consecuencia, creemos que esta última actitud, asi como a anterior, explicitan lo siguiente: el 75 se plantea una poítica lingüistica que aparecia en la superficie de un proyecto nacional reformista, que emergia de una consideración politica de la cuestión de la lengua y del problema de identidad, los cuales se manifestaban en el marco de la cultura y de un espacio nacional, no en base a la lengua en si o a las rivalidades de las lenguas, sino

(9) La impronta ideológica que marca los valores es digna de destacarse en el juicio de Alejandro $O$. Deustua, acerca de la disolución psíquica dé la raza indigena y la desgracia del Perú: "Es doloroso reconocer este hecho, pero es necesario reconocerlo para plantear el problema de la educación indigena dentro de los términos que la experiencia ofrece. Está bien que se utilice las habilidades mecánicas del indio; mucho mejor que se ampare $y$ defienda contra sus explotadores de todas espeais y que se introduzca en sus costumbres los hábitos de higiene que careze. Pero no debe irse más allá, sacrificando recursos que serán estériles en esa obra superior y que serian más provechosos en la satisfacción ur. gente de otras necesidades sociales. El indio no es $y$ no puede ser sino una máquina". La Cultura Nacional (1937). Cf. Alberti y Cotler (1972) señalan la contradicción en el sistema de relaciones sociales de tipo oligárquico, que por definición se fundaba en la exclusión de las masas populares de los mecanismos institucionales de la sociedad. 
merced al distinto rol entre los hispanohablantes maternos y los no hablantes de español, o no hablantes maternos de esta lengua, en grado o manera suficientemente aceptados. Por tanto, esta actitud tendia a enfatizar una relación social que ponia en relieve una de las formas de la discriminación que, curiosamente, tiene su impacto más extraordinario, en la carrera estudiantil que termina en los exámenes de ingreso a las universidades peruanas o en la búsqueda de acceso a un empleo. Si no se quiere hurgar tanto, para tener conciencia de lo que esto sígnifica en el proceso educativo del joven peruano, podría darse relieve a la tasa de deserción en el proceso escolar, tanto en el mundo urbano como en el rural $(10)$.

De los enunciados legales del 79 aparece de modo saltante el ánimo de diferenciar entre los que no dominan el español y los que asi lo hacen. Es decir, que aparece inmediatamente que los problemas de la lengua y de la identidad son colocados en un embolsamiento que apunta a la alfabetización y la educación hilingüe, y a una actitud proteccionista relegada a ulteriores fórmulas legales, si bien - por lo mismo- se consagra la alfabetización y la castellanización, a través de la lengua materna del educando. Pero lo más evidente es la restricción territorial por zonas o regiones, que generarian espacios cuyas fronteras cada vez son menos vis:bles, en la medida que la movilidad dentro del pais ha alcanzado, especialmente desde los años 50 , una dinámica que va tiñendo con distintos matices lo que hemos llamado la cuestión de la lengua en el Perú, así como en los países andinos.

Me inclino a pensar que no solamente hay una actitud proclive a ver en el problema lingüístico un típico hecho educativo (como antes de González Prada en 1904 o Mariátegui en 1928), o sea como lo vieran los pensadores que concibieron el Perú como una dicotomía entre la sierra y la costa, y para quienes la sı. tuación desarticulada en la sociedad peruana era equivalente a una falta "de progreso y civilización", a causa de la postración

(10) Para una muestra diremos que en 1975, la pirámide educacional arrojaba que de 7 estudiantes del primer grado solo 3 completaban el $f^{\circ}$ grado, y solo 1 terminaba la secundaria diurna. Cf. OSPE. Unidad de Estadistica, 1975, p. 15. 
del indio, el cual debería ser asimilado y educado. $Y$ para conseguir estos rsultados, la escuela se convertia en el centro irradiador de la politica integradora del Estado. Por cierto que estamos muy lejos de la posición opuesta, la cual, al contrario, carga el problema a la falta de éxito de la dirigencia para trazar un proyecto nacional compartible por las distintas clases sociales durante la república. Esta, desde los primeros años se ha empecinado en mantener una actitud colonial frente a las áreas interiores del pais, y en todo caso, no percibe que es el ámbito general del Estado el escenario $-y$ no sólo la escuela-, en el cual se define una relación asimétrica en lo social y en lo económico, que tiene, y no por azar, su contrapartida en la jerarquización de los roles y del prestigio de las lenguas que se emplean.

Finalmente, y teniendo en cuenta que el presente es un comentario sobre textos y antecedentes culturales, politicos, educativos y lingüísticos, con la explicable prudencia que es propia del debate académico, me permitiria presentar cuatro reflexiones.

1. Todo recuento de la política indigenista peruana está signado por un carácter impuesto por actores pequeño-burgueses. que, por lo general, han sido politicos o técnicos arraigados en la capital.

2. Descontado lo anterior, que es válido tanto para el 75 como para el 79 , lo que importa es el sentido histórico de las acciones imaginadas y la perspectiva de su aplicación. Es decir. si tienden a encubrir el problema social y económico, para en su lugar, convertirlo en un problema individual $y$ educativo.

3. Es revelador pensar que solamente son algunas regiones del Perú, que es casi como pensar en términos de las antiguas reducciones españolas, las que afrontan un problema lingüistico que tiene una serie de derivaciones en muchísimos campos de la vida nacional. De modo que hay notable diferencia entre entender toda esta cuestión desde un punto de vista regional o nacional. Piénsese especialmente en los alcances de la radio y de la televisión en los dias presentes, y en la avidez por llegar a los sectores más apartados del pais; pero, como siempre, para ofrecerles una palabra que tiene so- 
lamente un canal de ida pero no otro de vuelta, a fin de escuchar a quienes por lo común ni hablan, ni lo pueden hacer como las normas de la retórica y con la pronunciación que en Lima demandan los llamados sectores "educados". Este es un ejemplo muy vivo de cómo amplios sectores de la población peruana difícilmente pueden hacerse escuchar en términos figurados o en términos de comunicación lingüistica, política, artistica, cultural en suma.

4. Llegados a este punto, creo que los articulos de la Constitucoón no se distinguen por ser especialmente anti-colonialistas, pero que dejan la posibilidad de que las leyes que los reglamenten puedan amenguar el efecto discriminatorio que podria ser la resultante de su aplicación estricta. En fin, en mi opinión, este es uno de los cruceros que definen la estructura de la sociedad peruana $y$, por lo tanto, hay que habituarse a entenderlo como un planteo político, social y económico, antes que como un ejercicio de prácticas académicas o pedagógicas. 


\section{RERERENCIAS}

ALBERTI, Georgio y COTLER, Julio

1972 Aspectos sociales de la educación rural en el Perú. IEP. Lima.

BONILLA, Heraclio

1974 Guano y Burguesía en el Perú. IEP. Lima.

1981 et al. La independencia en el Perú. 2da. edc. IEP. Lima.

1982 "Etnia, región, y la cuestión nacional en el área andina". En: Indianité, ethnocidie, indigénisme en Amériques Latise. GRAL, C.N.R.S. Paris, pp. 59-77.

BURGA, Manuel y Alberto FLORES GALINDO

1979. Apogeo y crisis de la república aristocrática. Ed. Rikchay Perú. Lima.

COTLER, Julio

1978 Clases, estado y nación en el Perú. IEP. Lima.

ESCOBAR, Alberto

1972 "Lingüistica y Derecho". En: Lenguaje y discriminación social en América Latina. Milla Batres. Lima.

1975 "La educación bilingüe en el Perú (1974)". En: Proceedings of the First Inter-American "Conference on Bilingual Education. Edited by R.C. Troike and N. Modiano. Center for Applied Lingüistics. Arlington, Virginia, pp. 32-42.

1976 "Bilingualism and Dialectology in Peru", in International Journal of the Sociology of Language. Mouton. The Hague, pp. 85-96.

1978 Variaciones sociolingüisticas del castellano en el Perú. IEP. Lima

1981 "En torno de la oficialización del Quechua en el Perú". En: L'Indigénisme Andin: Approches, tendances et perspectives, C.E.R.P.A. Grenoble, pp. 236-240. 
1982 "Situación multilingüe y planeamiento lingüistico en el Perú", en: Indianité, ethnocide, indigénisme en Amérique Latine. GRAL, C.N.R.S. Paris, pp. 95-104.

GARCIA SALVATTECCI, Hugo

1972 (?) El pensamiento de González Prada. Ed. Arica. Lima.

GONZALEZ PRADA, Manuel

1978 Sobre el militarismo (Antologia) Bajo el oprobio. Selec. y presentación de B. PODESTA. Ed. Horizonte. Lima.

PODESTA, Bruno

1980 "La gestación del primer indigenismo: el caso de González Prada". En: L'Indigénisme Andin: Approches, tendances et pesrpectives. C.E.R.P.A. Grenoble, pp. 9-18.

1075 Pensamiento politico de Gonzälez Prada. INC. Lima, pp. 11-51. 